\title{
Fitting of Normal Distribution by Using Areas Method between Rainfall and Ground Water Levels - A Case Study
}

Raju Sake

Academic Consultant, Department of Statistics, Sri Krishnadevaraya University, Anantapuramu, (A.P), India.

E-mail: raju.hcu2007@gmail.com

DOI: http://doi.org/10.38177/ajast.2021.5407

Copyright: () 2021 Raju Sake. This is an open access article distributed under the terms of the Creative Commons Attribution License, which permits unrestricted use, distribution, and reproduction in any medium, provided the original author and source are credited.

\section{AB STR ACT}

Present paper deals with the application of 'Normal distribution' to analyze and predict Rainfall (RF) and Ground water levels (GWLs) in Anantapuramu district based on the data collected from January 2007 to December 2016. With Normal distribution by using areas method, for the purpose of analysis the district is divided into five zones or Revenue Divisions (RD) namely, (1) Anantapuramu RD (2) Penukonda RD (3) Kadiri RD (4) Kalyandurg RD (5) Dharmavaram RD. The values of Normal distribution have been calculated by using areas method and compared among them by using the data and conclusions are drawn based on the results obtained.

Keywords: Rainfall, Ground water level, Normal distribution, Areas method, Prediction.

\section{Introduction}

I have discussed 'Distribution Theory' for different distributions like. Binomial Distribution-Direct and Recurrence Relation Method, Negative Binomial Distribution Recurrence Relation Method and Poisson distribution direct and recurrence relation methods already I have analyzed; now in this paper, I will fit Normal distribution by using areas method.

The data is collected on Average Rainfall and Average Ground Water Levels are given in the following Table-1 for a ready reference $[1,2,3,4,5,6,7,8$ and 9$]$.

Table 1. Average Rainfall and Average Ground water levels data from 2007 to 2016

\begin{tabular}{|c|c|c|c|c|c|c|c|c|c|c|}
\hline \multirow{2}{*}{ Year } & \multicolumn{2}{|c|}{ Zone-I } & \multicolumn{2}{c|}{ Zone-II } & \multicolumn{2}{c|}{ Zone-III } & \multicolumn{2}{c|}{ Zone-IV } & \multicolumn{2}{c|}{ Zone-V } \\
\cline { 2 - 12 } & $\begin{array}{c}\text { RF } \\
\text { (in mm) }\end{array}$ & GWL & $\begin{array}{c}\text { RF } \\
\text { (in mm) }\end{array}$ & GWL & $\begin{array}{c}\text { RF } \\
\text { (in mm) }\end{array}$ & GWL & $\begin{array}{c}\text { RF } \\
\text { (in mm) }\end{array}$ & GWL & $\begin{array}{c}\text { RF } \\
\text { (in mm) }\end{array}$ & GWL \\
\hline 2007 & 65.60 & 10.57 & 58.20 & 22.58 & 67.20 & 14.23 & 52.00 & 14.97 & 60.50 & 17.03 \\
\hline 2008 & 53.90 & 9.96 & 77.90 & 20.73 & 65.20 & 9.27 & 61.30 & 10.88 & 62.70 & 9.09 \\
\hline 2009 & 45.40 & 12.17 & 50.60 & 17.53 & 46.30 & 11.08 & 57.10 & 9.58 & 38.70 & 10.24 \\
\hline 2010 & 53.90 & 12.74 & 71.50 & 15.02 & 70.80 & 12.03 & 64.60 & 8.58 & 56.30 & 11.79 \\
\hline 2011 & 39.50 & 12.69 & 42.30 & 15.20 & 48.90 & 11.48 & 31.80 & 8.93 & 36.60 & 12.84 \\
\hline 2012 & 43.20 & 14.98 & 43.40 & 20.49 & 45.30 & 16.08 & 40.50 & 13.76 & 41.90 & 13.22 \\
\hline 2013 & 35.00 & 15.94 & 52.30 & 23.03 & 47.10 & 18.69 & 34.80 & 16.98 & 38.10 & 14.30 \\
\hline 2014 & 31.10 & 15.87 & 30.30 & 23.40 & 27.10 & 21.16 & 37.10 & 18.92 & 22.80 & 16.30 \\
\hline 2015 & 44.10 & 14.90 & 62.60 & 26.88 & 66.30 & 25.80 & 46.00 & 19.26 & 54.30 & 17.66 \\
\hline 2016 & 33.50 & 15.57 & 33.40 & 27.27 & 32.30 & 15.35 & 25.70 & 19.51 & 30.10 & 16.15 \\
\hline
\end{tabular}




\section{Statistical Analysis}

To analyze Rainfall and Ground Water Levels through Normal distribution by using areas method for different zones we can consider given as follows:

The p.d.f. of Normal distribution is given by,

$f(x ; \mu, \sigma)=\frac{1}{\sigma \sqrt{2 \pi}} e^{\frac{-1}{2}\left(\frac{x-\mu}{\sigma}\right)^{2}} ;-\infty<x<\infty,-\infty<\mu<\infty, \sigma>0$

Here, $\mu$ and $\sigma$ are called the parameters of Normal distribution. These are estimated as,

$\bar{x}=\frac{\sum_{i=1}^{n} f_{i} x_{i}}{N=\sum_{i=1}^{n} f_{i}}=\hat{\mu}$

$\sigma^{2}=\frac{1}{N} f_{i} x_{i}^{2}-(\bar{x})^{2}=\hat{\sigma}^{2}$

Therefore, the p.d.f. of Normal distribution fitted to the given data is,

$f(x ; \hat{\mu}, \hat{\sigma})=\frac{1}{\hat{\sigma} \sqrt{2 \pi}} e^{\frac{-1}{2}\left(\frac{x-\hat{\mu}}{\hat{\sigma}}\right)^{2}} ;-\infty<x<\infty,-\infty<\hat{\mu}<\infty, \hat{\sigma}>0$

\section{To find the Expected Frequencies}

To find the Expected Frequencies, we use the following steps.

Step-I: We convert the given data into the continuous frequency distribution.

Step-II: The Standard Normal Variate $z$ is defined as,

$z_{i}=\frac{\left(x_{i}-\mu\right)}{\sigma}$

Where, $x_{i}$ are the lower limits of the class interval, the areas of each $z_{i}$ are taken from Normal tables, for the calculation of frequencies.

The fitted Normal distribution by using areas method for Average RF and Average GWLs:

\section{A: For Average Rainfall}

\section{Zone-I}

The p.d.f. of Normal distribution is given by,

$f(x ; \mu, \sigma)=\frac{1}{2.91 \sqrt{2 \pi}} e^{\frac{-1}{2}\left(\frac{x-4.96}{2.91}\right)^{2}} ;-\infty<x<\infty,-\infty<\mu<\infty, \sigma>0$

\begin{tabular}{|c|c|c|c|c|c|}
\hline C.I & Lower Limit & $z_{i}=\frac{\left(x_{i}-\mu\right)}{\sigma}$ & Areas $\phi\left(z_{i}\right)$ & $\Delta \times \phi\left(z_{i}\right)$ & $\begin{array}{c}\mathbf{N} \times\left(\Delta \times \phi\left(z_{i}\right)\right) \\
\text { frequencies }\end{array}$ \\
\hline$-\infty---0.5$ & $-\infty$ & $-\infty$ & 0.5 & \multirow{4}{*}{$\begin{array}{l}-0.06 \\
-0.05 \\
-0.08\end{array}$} & \multirow{4}{*}{$\begin{array}{r}-26.71 \\
-22.26 \\
-35.62\end{array}$} \\
\hline $0.5----1.5$ & 0.5 & -1.53 & 0.4370 & & \\
\hline $1.5----2.5$ & 1.5 & -1.19 & 0.3830 & & \\
\hline $2.5----3.5$ & 2.5 & -0.85 & 0.3023 & & \\
\hline
\end{tabular}


Asian Journal of Applied Science and Technology (AJAST)

Volume 5, Issue 4, Pages 55-64, Oct-Dec 2021

\begin{tabular}{|c|c|c|c|c|c|}
\hline $3.5----4.5$ & 3.5 & -0.50 & 0.1915 & -0.11 & -48.97 \\
\hline $4.5----5.5$ & 4.5 & -0.16 & 0.0636 & -0.13 & -57.88 \\
\hline $5.5---6.5$ & 5.5 & 0.19 & 0.0759 & 0.01 & 4.45 \\
\hline $6.5----7.5$ & 6.5 & 0.53 & 0.2019 & 0.13 & 57.88 \\
\hline $7.5----8.5$ & 7.5 & 0.87 & 0.3078 & 0.11 & 48.97 \\
\hline 8.5----9.5 & 8.5 & 1.22 & 0.3888 & 0.08 & 35.62 \\
\hline 9.5----10.5 & 9.5 & 1.56 & 0.4406 & 0.05 & 22.26 \\
\hline $10.5--+\infty$ & 10.5 & 1.90 & 0.4713 & 0.03 & 13.36 \\
\hline
\end{tabular}

\section{Zone-II}

The p.d.f. of Normal distribution is given by,

$f(x ; \mu, \sigma)=\frac{1}{2.86 \sqrt{2 \pi}} e^{\frac{-1}{2}\left(\frac{x-5.03}{2.86}\right)^{2}} ;-\infty<x<\infty,-\infty<\mu<\infty, \sigma>0$

\begin{tabular}{|c|c|c|c|c|c|}
\hline C.I & Lower Limit & $z_{i}=\frac{\left(x_{i}-\mu\right)}{\sigma}$ & $\operatorname{Areas} \phi\left(z_{i}\right)$ & $\Delta \times \phi\left(z_{i}\right)$ & $\begin{array}{c}\mathbf{N} \times\left(\Delta \times \phi\left(z_{i}\right)\right) \\
\text { frequencies }\end{array}$ \\
\hline$-\infty---0.5$ & $-\infty$ & $-\infty$ & 0.5 & & \\
\hline $0.5----1.5$ & 0.5 & -1.58 & 0.4429 & -0.06 & -31.35 \\
\hline $1.5----2.5$ & 1.5 & -1.23 & 0.3907 & -0.05 & -26.13 \\
\hline $2.5----3.5$ & 2.5 & -0.88 & 0.3106 & -0.08 & -41.80 \\
\hline $3.5----4.5$ & 3.5 & -0.53 & 0.2019 & -0.11 & -57.48 \\
\hline $4.5----5.5$ & 4.5 & -0.19 & 0.0759 & -0.13 & -67.93 \\
\hline 5.5----6.5 & 5.5 & 0.16 & 0.0636 & -0.01 & -5.23 \\
\hline $6.5----7.5$ & 6.5 & 0.51 & 0.1950 & 0.13 & 67.93 \\
\hline 7.5----8.5 & 7.5 & 0.86 & 0.3051 & 0.11 & 57.48 \\
\hline $8.5----9.5$ & 8.5 & 1.21 & 0.3869 & 0.08 & 41.80 \\
\hline 9.5----10.5 & 9.5 & 1.56 & 0.4406 & 0.05 & 26.13 \\
\hline $10.5--+\infty$ & 10.5 & 1.91 & 0.4719 & 0.03 & 15.68 \\
\hline
\end{tabular}

\section{Zone-III}

The p.d.f. of Normal distribution is given by,

$f(x ; \mu, \sigma)=\frac{1}{2.87 \sqrt{2 \pi}} e^{\frac{-1}{2}\left(\frac{x-5.04}{2.87}\right)^{2}} ;-\infty<x<\infty,-\infty<\mu<\infty, \sigma>0$

\begin{tabular}{|c|l|l|c|c|c|}
\hline C.I & Lower Limit & $z_{i}=\frac{\left(x_{i}-\mu\right)}{\sigma}$ & Areas $\phi\left(z_{i}\right)$ & $\Delta \times \phi\left(z_{i}\right)$ & $\begin{array}{c}\mathbf{N} \times\left(\Delta \times \phi\left(z_{i}\right)\right) \\
\text { frequencies }\end{array}$ \\
\hline$-\infty--0.5$ & $-\infty$ & $-\infty$ & 0.5 & & \\
\hline
\end{tabular}


Volume 5, Issue 4, Pages 55-64, Oct-Dec 2021

\begin{tabular}{|c|c|c|c|c|c|}
\hline $0.5----1.5$ & 0.5 & -1.58 & 0.4429 & -0.06 & -30.99 \\
\hline $1.5----2.5$ & 1.5 & -1.23 & 0.3907 & -0.05 & -25.83 \\
\hline $2.5---3.5$ & 2.5 & -0.89 & 0.3133 & -0.08 & -41.32 \\
\hline $3.5----4.5$ & 3.5 & -0.54 & 0.2054 & -0.11 & -56.82 \\
\hline $4.5----5.5$ & 4.5 & -0.19 & 0.0759 & -0.13 & -67.15 \\
\hline $5.5----6.5$ & 5.5 & 0.16 & 0.0636 & -0.01 & -5.17 \\
\hline $6.5---7.5$ & 6.5 & 0.51 & 0.1950 & 0.13 & 67.15 \\
\hline $7.5---8.5$ & 7.5 & 0.86 & 0.3051 & 0.11 & 56.82 \\
\hline 8.5----9.5 & 8.5 & 1.21 & 0.3869 & 0.08 & 41.32 \\
\hline 9.5----10.5 & 9.5 & 1.55 & 0.4394 & 0.05 & 25.83 \\
\hline $10.5--+\infty$ & 10.5 & 1.90 & 0.4713 & 0.03 & 15.50 \\
\hline
\end{tabular}

\section{Zone-IV}

The p.d.f. of Normal distribution is given by,

$f(x ; \mu, \sigma)=\frac{1}{2.81 \sqrt{2 \pi}} e^{\frac{-1}{2}\left(\frac{x-4.92}{2.81}\right)^{2}} ;-\infty<x<\infty,-\infty<\mu<\infty, \sigma>0$

\begin{tabular}{|c|c|c|c|c|c|}
\hline C.I & Lower Limit & $z_{i}=\frac{\left(x_{i}-\mu\right)}{\sigma}$ & $\operatorname{Areas} \phi\left(z_{i}\right)$ & $\Delta \times \phi\left(z_{i}\right)$ & $\begin{array}{c}\mathbf{N} \times\left(\Delta \times \phi\left(z_{i}\right)\right) \\
\text { frequencies }\end{array}$ \\
\hline$-\infty---0.5$ & $-\infty$ & $-\infty$ & 0.5 & & \\
\hline $0.5----1.5$ & 0.5 & -1.57 & 0.4418 & -0.06 & -27.05 \\
\hline $1.5----2.5$ & 1.5 & -1.22 & 0.3888 & -0.05 & -22.55 \\
\hline $2.5----3.5$ & 2.5 & -0.86 & 0.3051 & -0.08 & -36.07 \\
\hline $3.5----4.5$ & 3.5 & -0.51 & 0.1950 & -0.11 & -49.60 \\
\hline $4.5----5.5$ & 4.5 & -0.15 & 0.0596 & -0.14 & -63.13 \\
\hline $5.5----6.5$ & 5.5 & 0.21 & 0.0832 & 0.02 & 9.02 \\
\hline $6.5----7.5$ & 6.5 & 0.56 & 0.2123 & 0.13 & 58.62 \\
\hline $7.5----8.5$ & 7.5 & 0.92 & 0.3212 & 0.11 & 49.60 \\
\hline $8.5----9.5$ & 8.5 & 1.27 & 0.3980 & 0.08 & 36.07 \\
\hline 9.5----10.5 & 9.5 & 1.63 & 0.4484 & 0.05 & 22.55 \\
\hline $10.5--+\infty$ & 10.5 & 1.99 & 0.4767 & 0.03 & 13.53 \\
\hline
\end{tabular}

\section{Zone-V}

The p.d.f. of Normal distribution is given by,

$f(x ; \mu, \sigma)=\frac{1}{2.92 \sqrt{2 \pi}} e^{\frac{-1}{2}\left(\frac{x-4.98}{2.92}\right)^{2}} ;-\infty<x<\infty,-\infty<\mu<\infty, \sigma>0$ 
Asian Journal of Applied Science and Technology (AJAST)

Volume 5, Issue 4, Pages 55-64, Oct-Dec 2021

\begin{tabular}{|c|c|c|c|c|c|}
\hline C.I & Lower Limit & $z_{i}=\frac{\left(x_{i}-\mu\right)}{\sigma}$ & $\operatorname{Areas} \phi\left(z_{i}\right)$ & $\Delta \times \phi\left(z_{i}\right)$ & $\begin{array}{c}\mathbf{N} \times\left(\Delta \times \phi\left(z_{i}\right)\right) \\
\text { frequencies }\end{array}$ \\
\hline$-\infty---0.5$ & $-\infty$ & $-\infty$ & 0.5 & & \\
\hline $0.5----1.5$ & 0.5 & -1.53 & 0.4370 & -0.06 & -26.52 \\
\hline $1.5----2.5$ & 1.5 & -1.19 & 0.3830 & -0.05 & -22.10 \\
\hline $2.5----3.5$ & 2.5 & -0.85 & 0.3023 & -0.08 & -35.36 \\
\hline $3.5----4.5$ & 3.5 & -0.51 & 0.1950 & -0.11 & -48.62 \\
\hline $4.5----5.5$ & 4.5 & -0.16 & 0.0636 & -0.13 & -57.46 \\
\hline 5.5----6.5 & 5.5 & 0.18 & 0.0714 & 0.01 & 4.42 \\
\hline $6.5----7.5$ & 6.5 & 0.52 & 0.1985 & 0.13 & 57.46 \\
\hline $7.5----8.5$ & 7.5 & 0.86 & 0.3051 & 0.11 & 48.62 \\
\hline $8.5----9.5$ & 8.5 & 1.21 & 0.3869 & 0.08 & 35.36 \\
\hline 9.5----10.5 & 9.5 & 1.55 & 0.4394 & 0.05 & 22.10 \\
\hline $10.5--+\infty$ & 10.5 & 1.89 & 0.4706 & 0.03 & 13.26 \\
\hline
\end{tabular}

\section{B: For Average Ground water levels}

\section{Zone-I}

The p.d.f. of Normal distribution is given by,

$f(x ; \mu, \sigma)=\frac{1}{2.79 \sqrt{2 \pi}} e^{\frac{-1}{2}\left(\frac{x-5.91}{2.79}\right)^{2}} ;-\infty<x<\infty,-\infty<\mu<\infty, \sigma>0$

\begin{tabular}{|c|c|c|c|c|c|}
\hline C.I & Lower Limit & $z_{i}=\frac{\left(x_{i}-\mu\right)}{\sigma}$ & $\operatorname{Areas} \phi\left(z_{i}\right)$ & $\Delta \times \phi\left(z_{i}\right)$ & $\begin{array}{c}\mathbf{N} \times\left(\Delta \times \phi\left(z_{i}\right)\right) \\
\text { frequencies }\end{array}$ \\
\hline$-\infty---0.5$ & $-\infty$ & $-\infty$ & 0.5 & & \\
\hline $0.5----1.5$ & 0.5 & -1.94 & 0.4738 & -0.03 & -4.06 \\
\hline $1.5----2.5$ & 1.5 & -1.58 & 0.4429 & -0.03 & -4.06 \\
\hline $2.5----3.5$ & 2.5 & -1.22 & 0.3888 & -0.05 & -6.77 \\
\hline $3.5----4.5$ & 3.5 & -0.86 & 0.3051 & -0.08 & -10.83 \\
\hline $4.5----5.5$ & 4.5 & -0.51 & 0.1950 & -0.11 & -14.89 \\
\hline $5.5----6.5$ & 5.5 & -0.15 & 0.0596 & -0.14 & -18.95 \\
\hline $6.5----7.5$ & 6.5 & 0.21 & 0.0832 & 0.02 & 2.71 \\
\hline 7.5----8.5 & 7.5 & 0.57 & 0.2157 & 0.13 & 17.60 \\
\hline $8.5----9.5$ & 8.5 & 0.93 & 0.3238 & 0.11 & 14.89 \\
\hline 9.5----10.5 & 9.5 & 1.29 & 0.4015 & 0.08 & 10.83 \\
\hline $10.5--+\infty$ & 10.5 & 1.65 & 0.4505 & 0.05 & 6.77 \\
\hline
\end{tabular}




\section{Zone-II}

The p.d.f. of Normal distribution is given by,

$f(x ; \mu, \sigma)=\frac{1}{3.01 \sqrt{2 \pi}} e^{\frac{-1}{2}\left(\frac{x-5.84}{3.01}\right)^{2}} ;-\infty<x<\infty,-\infty<\mu<\infty, \sigma>0$

\begin{tabular}{|c|c|c|c|c|c|}
\hline C.I & Lower Limit & $z_{i}=\frac{\left(x_{i}-\mu\right)}{\sigma}$ & $\operatorname{Areas} \phi\left(z_{i}\right)$ & $\Delta \times \phi\left(z_{i}\right)$ & $\begin{array}{c}\mathbf{N} \times\left(\Delta \times \phi\left(z_{i}\right)\right) \\
\text { frequencies }\end{array}$ \\
\hline$-\infty---0.5$ & $-\infty$ & $-\infty$ & 0.5 & \multirow{3}{*}{-0.04} & \multirow[b]{2}{*}{-8.49} \\
\hline $0.5----1.5$ & 0.5 & -1.77 & 0.4616 & & \\
\hline $1.5----2.5$ & 1.5 & -1.44 & 0.4251 & & -8.49 \\
\hline $2.5----3.5$ & 2.5 & -1.11 & 0.3655 & -0.06 & -12.73 \\
\hline $3.5----4.5$ & 3.5 & -0.78 & 0.2823 & -0.08 & -16.97 \\
\hline $4.5----5.5$ & 4.5 & -0.45 & 0.1736 & \multirow{2}{*}{$\begin{array}{l}-0.11 \\
-0.13\end{array}$} & -23.33 \\
\hline $5.5----6.5$ & 5.5 & -0.11 & 0.0438 & & -27.58 \\
\hline $6.5----7.5$ & 6.5 & 0.22 & 0.0871 & 0.04 & 8.49 \\
\hline $7.5----8.5$ & 7.5 & 0.55 & 0.2088 & 0.12 & 25.46 \\
\hline $8.5----9.5$ & 8.5 & 0.88 & 0.3106 & \multirow{3}{*}{$\begin{array}{l}0.10 \\
0.08 \\
0.05\end{array}$} & 21.21 \\
\hline 9.5----10.5 & 9.5 & 1.22 & 0.3888 & & 16.97 \\
\hline $10.5--+\infty$ & 10.5 & 1.55 & 0.4394 & & 10.61 \\
\hline
\end{tabular}

\section{Zone-III}

The p.d.f. of Normal distribution is given by,

$f(x ; \mu, \sigma)=\frac{1}{2.82 \sqrt{2 \pi}} e^{\frac{-1}{2}\left(\frac{x-6.15}{2.82}\right)^{2}} ;-\infty<x<\infty,-\infty<\mu<\infty, \sigma>0$

\begin{tabular}{|c|c|c|c|c|c|}
\hline C.I & Lower Limit & $z_{i}=\frac{\left(x_{i}-\mu\right)}{\sigma}$ & $\operatorname{Areas} \phi\left(z_{i}\right)$ & $\Delta \times \phi\left(z_{i}\right)$ & $\begin{array}{c}\mathbf{N} \times\left(\Delta \times \phi\left(z_{i}\right)\right) \\
\text { frequencies }\end{array}$ \\
\hline$-\infty---0.5$ & $-\infty$ & $-\infty$ & 0.5 & & \\
\hline $0.5----1.5$ & 0.5 & -2.00 & 0.4772 & -0.02 & -3.10 \\
\hline $1.5----2.5$ & 1.5 & -1.65 & 0.4505 & -0.03 & -4.66 \\
\hline $2.5----3.5$ & 2.5 & -1.29 & 0.4015 & -0.05 & -7.76 \\
\hline $3.5----4.5$ & 3.5 & -0.94 & 0.3264 & -0.08 & -12.41 \\
\hline $4.5----5.5$ & 4.5 & -0.59 & 0.2224 & -0.10 & -15.52 \\
\hline $5.5----6.5$ & 5.5 & -0.23 & 0.0910 & -0.13 & -20.17 \\
\hline $6.5----7.5$ & 6.5 & 0.12 & 0.0478 & -0.04 & $-6,21$ \\
\hline $7.5----8.5$ & 7.5 & 0.48 & 0.1844 & 0.14 & 21.72 \\
\hline
\end{tabular}


Volume 5, Issue 4, Pages 55-64, Oct-Dec 2021

\begin{tabular}{|c|c|c|c|c|c|}
\hline $8.5----9.5$ & 8.5 & 0.83 & 0.2967 & 0.11 & 17.07 \\
\cline { 1 - 3 } $9.5----10.5$ & 9.5 & 1.19 & 0.3830 & 0.09 & 13.97 \\
\cline { 1 - 3 } $10.5--+\infty$ & 10.5 & 1.54 & 0.4382 & 0.06 & 9.31 \\
\hline
\end{tabular}

\section{Zone-IV}

The p.d.f. of Normal distribution is given by,

$f(x ; \mu, \sigma)=\frac{1}{2.98 \sqrt{2 \pi}} e^{\frac{-1}{2}\left(\frac{x-6.12}{2.98}\right)^{2}} ;-\infty<x<\infty,-\infty<\mu<\infty, \sigma>0$

\begin{tabular}{|c|c|c|c|c|c|}
\hline C.I & Lower Limit & $z_{i}=\frac{\left(x_{i}-\mu\right)}{\sigma}$ & $\operatorname{Areas} \phi\left(z_{i}\right)$ & $\Delta \times \phi\left(z_{i}\right)$ & $\begin{array}{c}\mathrm{N} \times\left(\Delta \times \phi\left(z_{i}\right)\right) \\
\text { frequencies }\end{array}$ \\
\hline$-\infty---0.5$ & $-\infty$ & $-\infty$ & 0.5 & & \\
\hline $0.5----1.5$ & 0.5 & -1.89 & 0.4706 & -0.03 & -4.24 \\
\hline $1.5----2.5$ & 1.5 & -1.55 & 0.4394 & -0.03 & -4.24 \\
\hline $2.5----3.5$ & 2.5 & -1.21 & 0.3869 & -0.05 & -7.07 \\
\hline $3.5----4.5$ & 3.5 & -0.88 & 0.3106 & -0.08 & -11.31 \\
\hline $4.5----5.5$ & 4.5 & -0.54 & 0.2054 & -0.11 & -15.55 \\
\hline $5.5----6.5$ & 5.5 & -0.21 & 0.0832 & -0.12 & -16.96 \\
\hline $6.5----7.5$ & 6.5 & 0.13 & 0.0517 & -0.03 & -4.24 \\
\hline $7.5----8.5$ & 7.5 & 0.46 & 0.1772 & 0.13 & 18.38 \\
\hline $8.5----9.5$ & 8.5 & 0.80 & 0.2881 & 0.11 & 15.55 \\
\hline 9.5----10.5 & 9.5 & 1.13 & 0.3708 & 0.08 & 11.31 \\
\hline $10.5-+\infty$ & 10.5 & 1.47 & 0.4292 & 0.06 & 8.48 \\
\hline
\end{tabular}

\section{Zone-V}

The p.d.f. of Normal distribution is given by,

$f(x ; \mu, \sigma)=\frac{1}{2.95 \sqrt{2 \pi}} e^{\frac{-1}{2}\left(\frac{x-5.83}{2.95}\right)^{2}} ;-\infty<x<\infty,-\infty<\mu<\infty, \sigma>0$

\begin{tabular}{|c|c|c|c|c|c|}
\hline C.I & Lower Limit & $z_{i}=\frac{\left(x_{i}-\mu\right)}{\sigma}$ & $\operatorname{Areas} \phi\left(z_{i}\right)$ & $\Delta \times \phi\left(z_{i}\right)$ & $\begin{array}{c}\mathbf{N} \times\left(\Delta \times \phi\left(z_{i}\right)\right) \\
\text { frequencies }\end{array}$ \\
\hline$-\infty--0.5$ & $-\infty$ & $-\infty$ & 0.5 & & \\
\hline $0.5---1.5$ & 0.5 & -1.81 & 0.4649 & -0.04 & -5.54 \\
\hline $1.5----2.5$ & 1.5 & -1.47 & 0.4292 & -0.04 & -5.54 \\
\hline $2.5----3.5$ & 2.5 & -1.13 & 0.3708 & -0.06 & -8.32 \\
\hline $3.5----4.5$ & 3.5 & -0.79 & 0.2852 & -0.09 & -12.48 \\
\hline $4.5----5.5$ & 4.5 & -0.45 & 0.1736 & -0.11 & -15.25 \\
\hline
\end{tabular}


Asian Journal of Applied Science and Technology (AJAST)

Volume 5, Issue 4, Pages 55-64, Oct-Dec 2021

\begin{tabular}{|c|c|c|c|c|c|}
\hline $5.5----6.5$ & 5.5 & -0.11 & 0.0438 & -0.13 & -18.02 \\
\hline $6.5----7.5$ & 6.5 & 0.23 & 0.0910 & 0.05 & 6.93 \\
\hline $7.5----8.5$ & 7.5 & 0.57 & 0.2157 & 0.12 & 16.63 \\
\hline $8.5----9.5$ & 8.5 & 0.91 & 0.3186 & 0.10 & 13.86 \\
\hline 9.5----10.5 & 9.5 & 1.24 & 0.3925 & 0.07 & 9.70 \\
\hline $10.5-+\infty$ & 10.5 & 1.58 & 0.4429 & 0.05 & 6.93 \\
\hline
\end{tabular}

Fig.1. Behavior of Avg RF and Avg GWLs frequencies for Normal distribution by using areas method in Zone-I, II, III, IV and V

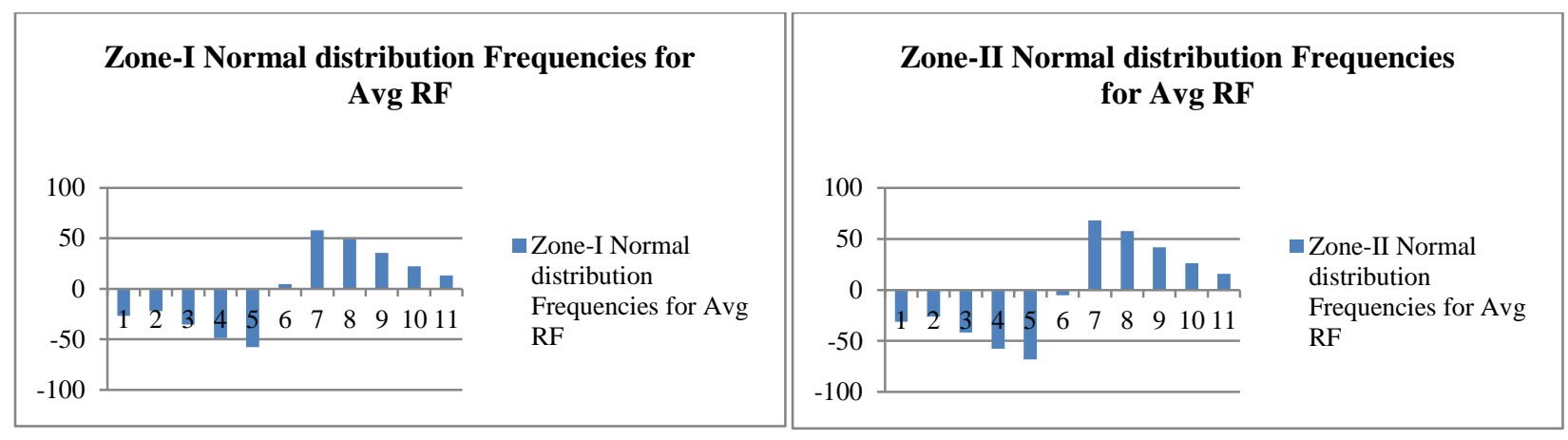

Zone-III Normal distribution Frequencies for Avg RF

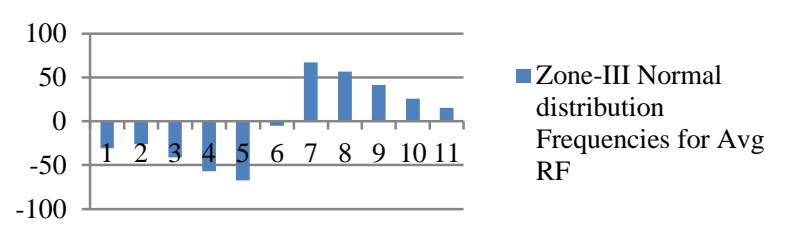

Zone-V Normal distribution Frequencies for Avg RF

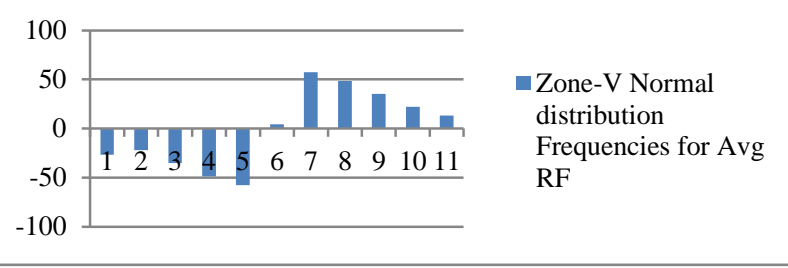

Zone-II Normal distribution Frequencies for Avg GWLs

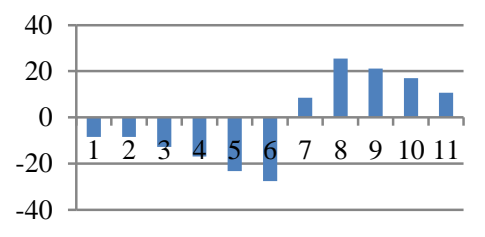

Zone-II Normal distribution

Frequencies for Avg GWLs
Zone-IV Normal distribution Frequencies for Avg RF

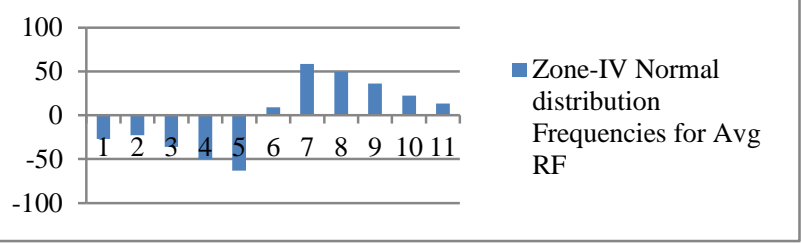

Zone-I Normal distribution Frequencies for Avg GWLs

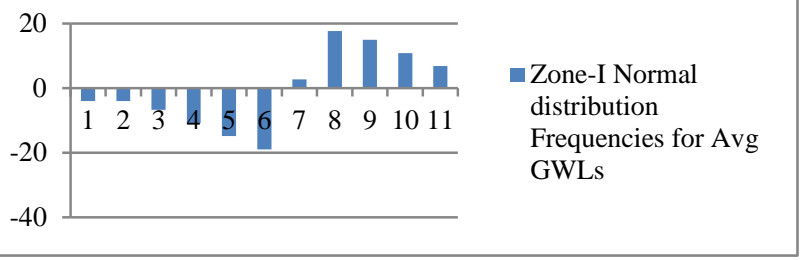

\section{Zone-III Normal distribution Frequencies for Avg GWLs}

$-3.1-4.66-7.76-12.41-15.52-20.17-6,21$

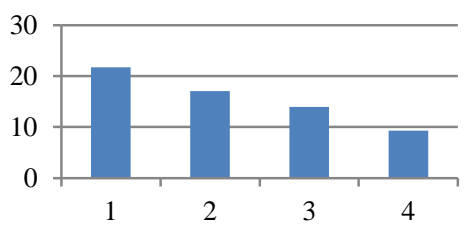

Zone-III Normal distribution Frequencies for Avg GWLs

$-3.1-4.66-7.76-$ $12.41-15.52-20.17$ 6,21 


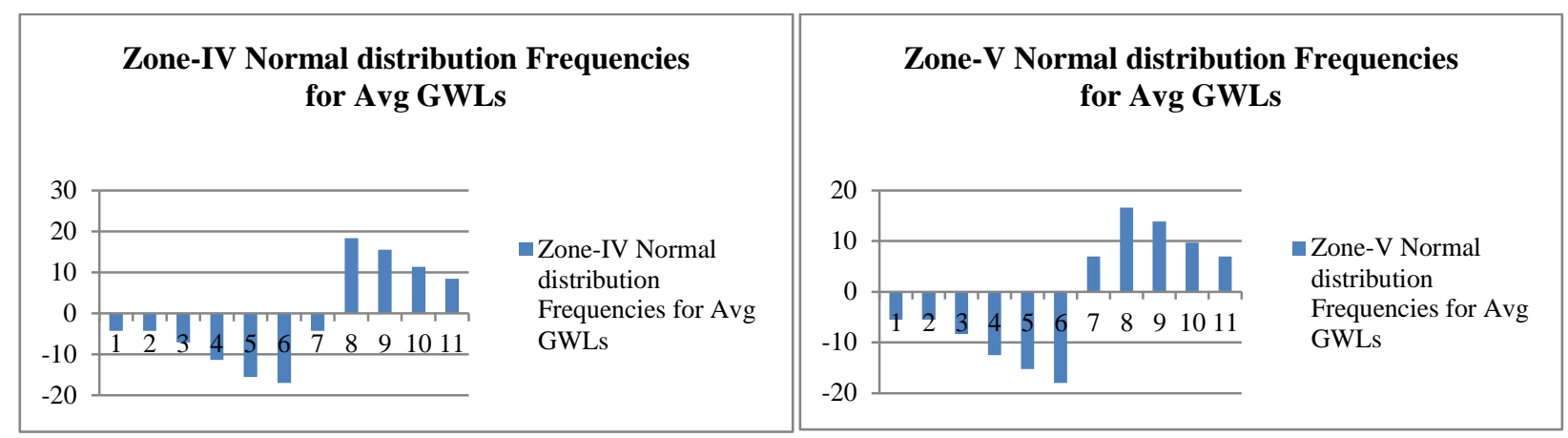

Note: Average RF measured in Mille Meters and Average GWLs measured in Meters.

\section{Conclusion}

By Comparing Average RF and Average GWLs frequencies through Normal distribution by using areas method under consideration, for Average RF and Average GWLs frequencies follows Normal distribution clearly as shown in the above fig. 1 .

\section{Declarations}

\section{Source of Funding}

This research did not receive any grant from funding agencies in the public, commercial, or not-for-profit sectors.

\section{Competing Interests Statement}

The author declares no competing financial, professional and personal interests.

\section{Consent for publication}

Author declares that he/she consented for the publication of this research work.

\section{Acknowledgements}

The author expresses heartfelt thanks to the persons responsible for giving the necessary data on Rainfall and Ground Water Levels working in Ground Water and Water Audit Department and Chief Planning Office Anantapuramu. Further the author is profusely thankful to Late Retd. Prof. K.L.A.P. Sarma, in the Department of Statistics, S.K. University, Anantapuramu. Mr. C. Pothulaiah, Asst. Hydrogeologist, Ground Water and Water Audit Department and Mr. Muralimohan Reddy, Assistant Statistical Officer, Chief Planning Office, Anantapurmau. Mr. Madhav Reddy, Scientific Assistant PBO Anantapuramu Meteorological Department and also Mr. N.V. Subbaraju, Mr. Lanka Ramakrishna Prasad working as a Computer Faculty and finally Mr. Muralikrishna from Jaipur in Rajasthan (State) for his timely suggestions and useful discussions. Further I expression sincere thanks to Sri Krishnadevaraya University authorities for providing me the necessary facilities in the Department, to complete my Research work [5, 6 and 7].

\section{References}

1. S. Raju, P. Mohammed Akhtar, "Time Series Analysis on Rainfall and Ground Water Levels Data - A Case Study", International Journal of Scientific Research in Mathematical and Statistical Sciences (IJSRMSS) Vol.6, Issue.1, pp.76-85, February (2019). 
2. S. Raju, P. Mohammed Akhtar, "Fitting of modified exponential model between rainfall and ground water levels: A case study", International Journal of Statistics and Applied Mathematics 2019; 4(4), pp. 01-06.

3. S. Raju, P. Mohammed Akhtar, "Fitting Of Gompertz Model Between Rainfall And Ground Water Levels - A Case Study", International Journal of Mathematics Trends and Technology (IJMTT) - Volume 65, Issue 7 - July (2019), pp. 85-93.

4. S. Raju, P. Mohammed Akhtar, "Fitting of Logistic Model between Rainfall and Ground Water Levels - A Case Study”, Compliance Engineering Journal, Volume 10, Issue 9, pp. 114-122, September-2019.

5. S. Raju, "Fitting of Poisson distribution by using recurrence relation method between Rainfall and Ground water levels - A Case Study", International Journal in Physical and Applied Sciences (IJPAS), Volume 7, Issue 1, January, 2020. pp. 76-87, (ISSN: 2394-5710).

6. S. Raju, "Fitting of binomial distribution by using recurrence relation method between rainfall and ground water levels:A case study", Journal of Mathematical Problems, Equations and Statistics (JMPES), Volume 1, Issue 02, pp. 03-08, 01-07-2020.

7. S. Raju, "Fitting of Modified Exponential Model between Rainfall and Ground Water Levels by Using Partial Sums Method - A Case Study”, Pensee Journal, Volume 51, Issue 03, pp. 647-655, March-2021.

8. S. Raju, P. Mohammed Akhtar, "Fitting of Gompertz model between Rainfall and Ground water levels by using Partial sums method - A case study”, Stochastic Modeling \& Applications, Vol. 25 No.1 (January-June, 2021), MuK Publications and Distributions (India), ISSN: 0972-3641.

9. S. Raju, "Fitting of Binomial Distribution between Rainfall and Ground Water Levels - A Case Study", International Journal of Engineering, Science and Mathematics (IJESM), (ISSN: 2320-0294).

10. S. Raju, "Fitting of Poisson distribution between Rainfall and Ground water levels - A Case Study", International Journal of Engineering\& Scientific Research (IJESR), Vol.9, Issue 6, June 2021, (ISSN: 2347-6532).

11. S. Raju, "Fitting of Negative Binomial Distribution by using Recurrence Relation Method between Rainfall and Ground Water Levels - A Case Study”, PARIPEX - Indian Journal of Research (PIJR), (ISSN: 2250-1991).

\section{Author Profile}

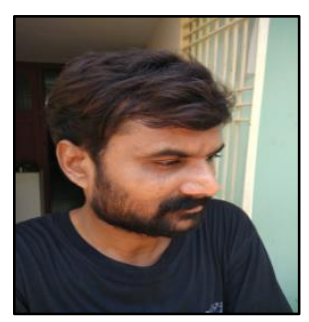

Raju Sake was awarded PhD degree in Statistics under the Supervision of Retd. Prof. P. Mohammed Akhtar in the Department of Statistics, S. K. University, Anantapuramu and Currently working as an Academic Consultant in the Department of Statistics, S.K. University, Anantapuramu, Andhra Pradesh, India. 